\title{
ON OPTIMIZATION OF MANUFACTURING PLANAR DOUBLE-BASE HETEROTRANSIS- TORS TO DECREASE THEIR DIMENSIONS
}

\author{
E.L. Pankratov ${ }^{1}$, E.A. Bulaeva ${ }^{1,2}$ \\ ${ }^{1}$ Nizhny Novgorod State University, 23 Gagarin avenue, Nizhny Novgorod, 603950, \\ Russia \\ ${ }^{2}$ Nizhny Novgorod State University of Architecture and Civil Engineering, 65 Il'insky \\ street, Nizhny Novgorod, 603950, Russia
}

\begin{abstract}
In this paper we consider an approach of manufacturing of double-base heterotransistors to decrease their dimensions. Framework the approach it should be manufactured a heterostructure with specific configuration. Farther it is necessary to dope certain areas of the heterostructure by diffusion or by ion implantation. After finishing of the doping process the dopant and/or radiation defects should be annealed. We consider an approach of optimization of dopant and/or radiation defects for manufacturing more compact doublebase heterotransistors.
\end{abstract}

\section{KEYWORDS}

Field-effect heterotransistors; double-base heterotransistors; increasing of integration rate

\section{INTRODUCTION}

Development of the solid state electronic devices leads to necessity to decrease dimensions of elements of integrated circuits, to increase their performance and reliability. One way to increase performance of the solid state electronic devices is searching materials with higher charge carriers mobility [1-3]. Another way to increase the performance is development of new or optimization of existing technological processes [4-7]. To increase reliability of the solid state electronic devices are could be also used development of new or optimization of existing technological processes [4-7]. One way to decrease dimensions of elements of integrated circuits is using laser or microwave types of annealing [8-10]. Using these types of annealing leads to generation inhomogenous distribution of temperature. The inhomogeneity with account Arrhenius law leads to inhomogeneity of dopant and radiation defects diffusion coefficients and another parameters. The inhomogeneity of parameters gives us possibility to decrease dimensions of elements of integrated circuits. Properties of materials could be also changed by using radiation processing $[11,12]$.

In this paper we consider a heterostructure, which consist of a substrate and epitaxial layer (see Figs. 1). The epitaxial layer includes into itself several sections, which manufactured by using another materials (see Figs. 1). The sections should be doped by diffusion or by ion implantation to generation required type of conductivity ( $n$ or $p$ ). We assume, that another area of epitaxial layer has reverse type of conductivity ( $p$ or $n$ ). We also assume, that substrate is insulator. Farther we consider annealing dopant and /or radiation defects. Increasing of annealing time leads to in- 
creasing of diffusion length into nearest materials. Decreasing of annealing time did not lead to full doping of sections of the epitaxial layer. Main aim of the present paper is choosing compromise value of annealing time.

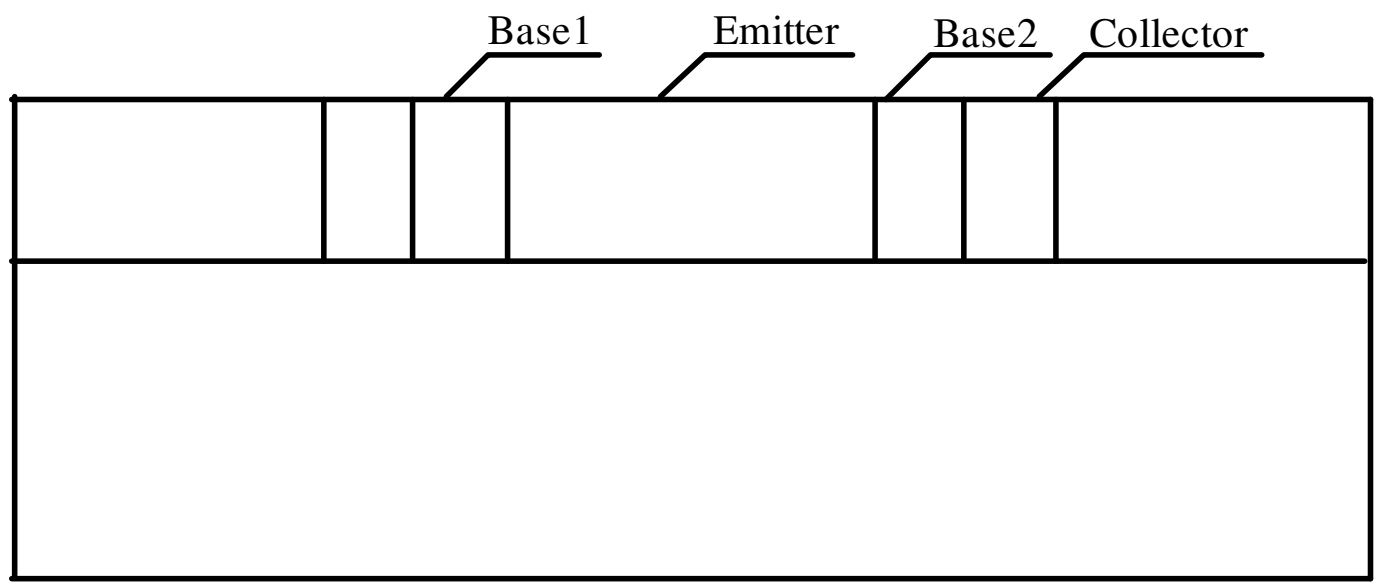

Fig. 1a. Heterostructure which consist of a substrate and a multisectional epitaxial layer. Side view

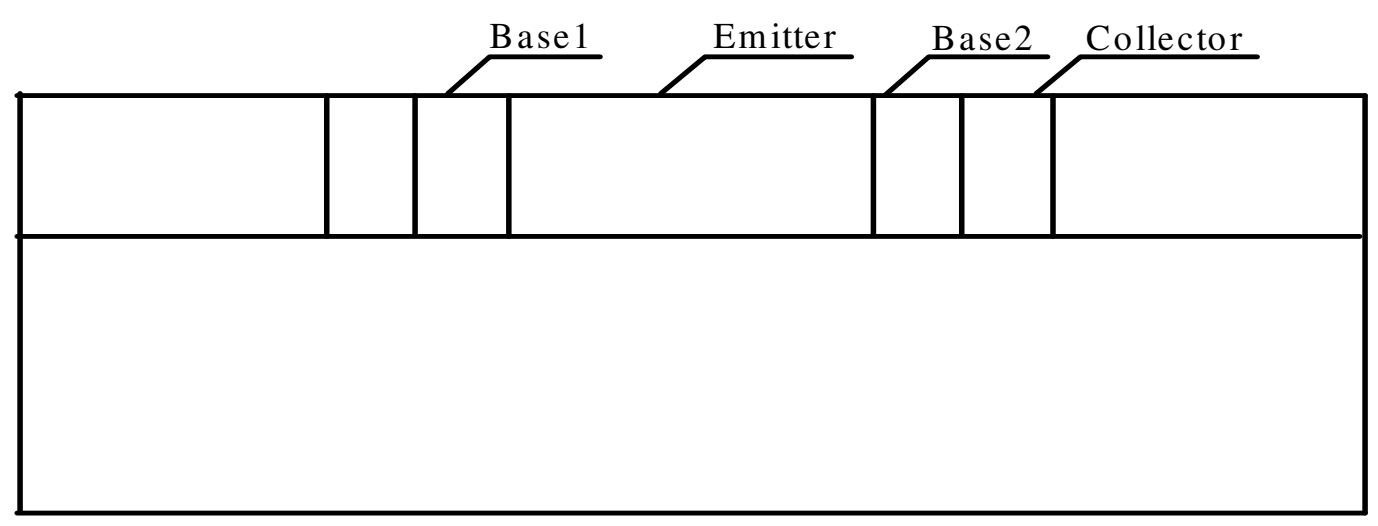

Fig.1b. Heterostructure which consist of a substrate and a multisectional epitaxial layer. Top view

\section{METHOD OF SOLUTION}

To achieve our aim we determine spatio-temporal distributions of concentrations of dopant and radiation defects. We determine the required distribution of concentrations of dopant by solution of the following boundary problem

$$
\begin{gathered}
\frac{\partial C(x, y, z, t)}{\partial t}=\frac{\partial}{\partial x}\left[D_{C} \frac{\partial C(x, y, z, t)}{\partial x}\right]+\frac{\partial}{\partial y}\left[D_{C} \frac{\partial C(x, y, z, t)}{\partial y}\right]+\frac{\partial}{\partial z}\left[D_{C} \frac{\partial C(x, y, z, t)}{\partial z}\right] \\
\left.\frac{\partial C(x, y, z, t)}{\partial x}\right|_{x=0}=0,\left.\frac{\partial C(x, y, z, t)}{\partial x}\right|_{x=L_{x}}=0,\left.\frac{\partial C(x, y, z, t)}{\partial y}\right|_{y=0}=0,\left.\frac{\partial C(x, y, z, t)}{\partial y}\right|_{x=L_{y}}=0 \\
\left.\frac{\partial C(x, y, z, t)}{\partial z}\right|_{z=0}=0,\left.\frac{\partial C(x, y, z, t)}{\partial z}\right|_{x=L_{z}}=0, C(x, y, z, 0)=f(x, y, z) .
\end{gathered}
$$


Here $C(x, y, z, t)$ is the spatio-temporal distribution of concentration of dopant; $T$ is the temperature of annealing; $D_{C}$ is the dopant diffusion coefficient. Value of dopant diffusion coefficient depends on properties of materials of heterostructure, speed of heating and cooling of heterostructure (with account Arrhenius law). Dependences of dopant diffusion coefficient on parameters could be approximated by the following relation [12-14]

$$
D_{C}=D_{L}(x, y, z, T)\left[1+\xi \frac{C^{\gamma}(x, y, z, t)}{P^{\gamma}(x, y, z, T)}\right]\left[1+\varsigma_{1} \frac{V(x, y, z, t)}{V^{*}}+\varsigma_{2} \frac{V^{2}(x, y, z, t)}{\left(V^{*}\right)^{2}}\right] .
$$

where $D_{L}(x, y, z, T)$ is the spatial (due to presents several layers in heterostructure) and temperature (due to Arrhenius law) dependences of dopant diffusion coefficient; $P(x, y, z, T)$ is the limit of solubility of dopant; parameter $\gamma$ depends on properties of materials and could be integer in the following interval $\gamma \in[1,3][13] ; V(x, y, z, t)$ is the spatio-temporal distribution of concentration of radiation vacancies; $V^{*}$ is the equilibrium distribution of concentration of vacancies. Concentrational dependence of dopant diffusion coefficient has been described in details in [13]. It should be noted, that using diffusion type of doping did not generation radiation defects. In this situation $\zeta_{1}=\zeta_{2}=0$. We determine spatio-temporal distributions of concentrations of radiation defects by solving the following boundary problem $[12,14]$

$$
\begin{aligned}
& \frac{\partial I(x, y, z, t)}{\partial t}=\frac{\partial}{\partial x}\left[D_{I}(x, y, z, T) \frac{\partial I(x, y, z, t)}{\partial x}\right]+\frac{\partial}{\partial y}\left[D_{I}(x, y, z, T) \frac{\partial I(x, y, z, t)}{\partial y}\right]-k_{I, I}(x, y, z, T) \times \\
& \quad \times I^{2}(x, y, z, t)+\frac{\partial}{\partial z}\left[D_{I}(x, y, z, T) \frac{\partial I(x, y, z, t)}{\partial z}\right]-k_{I, V}(x, y, z, T) I(x, y, z, t) V(x, y, z, t) \quad(4) \\
& \frac{\partial V(x, y, z, t)}{\partial t}=\frac{\partial}{\partial x}\left[D_{V}(x, y, z, T) \frac{\partial V(x, y, z, t)}{\partial x}\right]+\frac{\partial}{\partial y}\left[D_{V}(x, y, z, T) \frac{\partial V(x, y, z, t)}{\partial y}\right]-k_{V, V}(x, y, z, T) \times \\
& \quad \times V^{2}(x, y, z, t)+\frac{\partial}{\partial z}\left[D_{V}(x, y, z, T) \frac{\partial V(x, y, z, t)}{\partial z}\right]-k_{I, V}(x, y, z, T) I(x, y, z, t) V(x, y, z, t) .
\end{aligned}
$$

Boundary and initial conditions for these equations are

$$
\begin{aligned}
\left.\frac{\partial \rho(x, y, z, t)}{\partial x}\right|_{x=0} & =0,\left.\frac{\partial \rho(x, y, z, t)}{\partial x}\right|_{x=L_{x}}=0,\left.\frac{\partial \rho(x, y, z, t)}{\partial y}\right|_{y=0}=0,\left.\frac{\partial \rho(x, y, z, t)}{\partial y}\right|_{y=L_{y}}=0, \\
& \left.\frac{\partial \rho(x, y, z, t)}{\partial z}\right|_{z=0}=0,\left.\frac{\partial \rho(x, y, z, t)}{\partial z}\right|_{z=L_{z}}=0, \rho(x, y, z, 0)=f_{\rho}(x, y, z) .
\end{aligned}
$$

Here $\rho=I, V ; I(x, y, z, t)$ is the spatio-temporal distribution of concentration of radiation interstitials; $D_{\rho}(x, y, z, T)$ are the diffusion coefficients of point radiation defects; terms $V^{2}(x, y, z, t)$ and $I^{2}(x, y, z, t)$ correspond to generation divacancies and diinterstitials; $k_{I, V}(x, y, z, T)$ is the parameter of recombination of point radiation defects; $k_{I, I}(x, y, z, T)$ and $k_{V, V}(x, y, z, T)$ are the parameters of generation of simplest complexes of point radiation defects. 
We determine spatio-temporal distributions of concentrations of divacancies $\Phi_{V}(x, y, z, t)$ and diinterstitials $\Phi_{I}(x, y, z, t)$ by solving the following boundary problem $[12,14]$

$$
\begin{gathered}
\frac{\partial \Phi_{I}(x, y, z, t)}{\partial t}=\frac{\partial}{\partial x}\left[D_{\Phi I}(x, y, z, T) \frac{\partial \Phi_{I}(x, y, z, t)}{\partial x}\right]+\frac{\partial}{\partial y}\left[D_{\Phi I}(x, y, z, T) \frac{\partial \Phi_{I}(x, y, z, t)}{\partial y}\right]+ \\
+\frac{\partial}{\partial z}\left[D_{\Phi I}(x, y, z, T) \frac{\partial \Phi_{I}(x, y, z, t)}{\partial z}\right]+k_{I, I}(x, y, z, T) I^{2}(x, y, z, t)-k_{I}(x, y, z, T) I(x, y, z, t) \\
\frac{\partial \Phi_{V}(x, y, z, t)}{\partial t}=\frac{\partial}{\partial x}\left[D_{\Phi V}(x, y, z, T) \frac{\partial \Phi_{V}(x, y, z, t)}{\partial x}\right]+\frac{\partial}{\partial y}\left[D_{\Phi V}(x, y, z, T) \frac{\partial \Phi_{V}(x, y, z, t)}{\partial y}\right]+ \\
+\frac{\partial}{\partial z}\left[D_{\Phi V}(x, y, z, T) \frac{\partial \Phi_{V}(x, y, z, t)}{\partial z}\right]+k_{V, V}(x, y, z, T) V^{2}(x, y, z, t)-k_{V}(x, y, z, T) V(x, y, z, t), \\
\left.\frac{\partial \Phi_{\rho}(x, y, z, t)}{\partial x}\right|_{x=0}=0,\left.\frac{\partial \Phi_{\rho}(x, y, z, t)}{\partial x}\right|_{x=L_{x}}=0,\left.\frac{\partial \Phi_{\rho}(x, y, z, t)}{\partial y}\right|_{y=0}=0,\left.\frac{\partial \Phi_{\rho}(x, y, z, t)}{\partial y}\right|_{y=L_{y}}=0, \\
\left.\frac{\partial \Phi_{\rho}(x, y, z, t)}{\partial z}\right|_{z=0}=0,\left.\frac{\partial \Phi_{\rho}(x, y, z, t)}{\partial z}\right|_{z=L_{z}}=0, \Phi_{I}(x, y, z, 0)=f_{\Phi I}(x, y, z), \Phi_{V}(x, y, z, 0)=f_{\Phi V}(x, y, z) .(7)
\end{gathered}
$$

Here $D_{\Phi \rho}(x, y, z, T)$ are the diffusion coefficients of the above complexes of radiation defects; $k_{I}(x, y, z, T)$ and $k_{V}(x, y, z, T)$ are the parameters of decay of these complexes.

We determine spatio-temporal distribution of concentrations of dopant and radiation defects by method of averaging of function corrections [15] with decreasing quantity of iteration steps [16]. Framework the approach we used solutions of Eqs. (1), (4), (6) with averaged values of diffusion coefficients $D_{0 L}, D_{0 I}, D_{0 V}, D_{0 \Phi I}, D_{0 \Phi V}$, zero values of parameters of recombination of radiation defects and parameters of generation and decay of their complexes as initial-order approximations of required concentrations. The initial-order approximations could be written as

$$
\begin{gathered}
C_{1}(x, y, z, t)=\frac{1}{L_{x} L_{y} L_{z}}+\frac{2}{L_{x} L_{y} L_{z}} \sum_{n=1}^{\infty} F_{n C} c_{n}(x) c_{n}(y) c_{n}(z) e_{n C}(t), \\
I_{1}(x, y, z, t)=\frac{1}{L_{x} L_{y} L_{z}}+\frac{2}{L_{x} L_{y} L_{z}} \sum_{n=1}^{\infty} F_{n I} c_{n}(x) c_{n}(y) c_{n}(z) e_{n I}(t), \\
V_{1}(x, y, z, t)=\frac{1}{L_{x} L_{y} L_{z}}+\frac{2}{L_{x} L_{y} L_{z}} \sum_{n=1}^{\infty} F_{n V} c_{n}(x) c_{n}(y) c_{n}(z) e_{n V}(t), \\
\Phi_{I 1}(x, y, z, t)=\frac{1}{L_{x} L_{y} L_{z}}+\frac{2}{L_{x} L_{y} L_{z}} \sum_{n=1}^{\infty} F_{n \Phi_{I}} c_{n}(x) c_{n}(y) c_{n}(z) e_{n \Phi_{I}}(t), \\
\Phi_{V 1}(x, y, z, t)=\frac{1}{L_{x} L_{y} L_{z}}+\frac{2}{L_{x} L_{y} L_{z}} \sum_{n=1}^{\infty} F_{n \Phi_{V}} c_{n}(x) c_{n}(y) c_{n}(z) e_{n \Phi_{V}}(t),
\end{gathered}
$$


where $e_{n \rho}(t)=\exp \left[-\pi^{2} n^{2} D_{0 \rho} t\left(\frac{1}{L_{x}^{2}}+\frac{1}{L_{y}^{2}}+\frac{1}{L_{z}^{2}}\right)\right], \quad F_{n \rho}=\int_{0}^{L_{x}} c_{n}(u) \int_{0}^{L_{y}} c_{n}(v) \int_{0}^{L_{z}} c_{n}(w) f_{\rho}(u, v, w) d w d v d u$, $c_{n}(\chi)=\cos \left(\pi n \chi / L_{\chi}\right)$.

We determine approximations of the second-and higher-orders framework standard iteration procedure of method of averaging of function corrections [16,17]. Framework this procedure to determine the $n$-th-order approximation of concentrations of dopant and radiation defects we shall replace the required functions $C(x, y, z, t), I(x, y, z, t), V(x, y, z, t), \Phi_{I}(x, y, z, t)$ and $\Phi_{V}(x, y, z, t)$ in the right sides of Eqs. (1), (4), (6) on the following sums $\alpha_{n}+\rho_{n-1}(x, y, z, t)$, when $\alpha_{n \rho}$ are not yet known average values of the $n$-th-order approximation of the above concentrations. The replacement leads to the following relations for the second-order approximations of the required concentrations

$$
\begin{aligned}
& \frac{\partial C_{2}(x, y, z, t)}{\partial t}=\frac{\partial}{\partial x}\left(D_{L}(x, y, z, T)\left[1+\varsigma_{1} \frac{V(x, y, z, t)}{V^{*}}+\varsigma_{2} \frac{V^{2}(x, y, z, t)}{\left(V^{*}\right)^{2}}\right] \frac{\partial C_{1}(x, y, z, t)}{\partial x} \times\right. \\
& \left.\times\left\{1+\xi \frac{\left[\alpha_{2 C}+C_{1}(x, y, z, t)\right]^{\gamma}}{P^{\gamma}(x, y, z, T)}\right\}\right)+\frac{\partial}{\partial y}\left(D_{L}(x, y, z, T)\left[1+\varsigma_{1} \frac{V(x, y, z, t)}{V^{*}}+\varsigma_{2} \frac{V^{2}(x, y, z, t)}{\left(V^{*}\right)^{2}}\right] \times\right. \\
& \left.\times\left\{1+\xi \frac{\left[\alpha_{2 C}+C_{1}(x, y, z, t)\right]^{\gamma}}{P^{\gamma}(x, y, z, T)}\right\} \frac{\partial C_{1}(x, y, z, t)}{\partial y}\right)+\frac{\partial}{\partial z}\left(\left\{1+\xi \frac{\left[\alpha_{2 C}+C_{1}(x, y, z, t)\right]^{\gamma}}{P^{\gamma}(x, y, z, T)}\right\} \times\right. \\
& \left.\times D_{L}(x, y, z, T)\left[1+\varsigma_{1} \frac{V(x, y, z, t)}{V^{*}}+\varsigma_{2} \frac{V^{2}(x, y, z, t)}{\left(V^{*}\right)^{2}}\right] \frac{\partial C_{1}(x, y, z, t)}{\partial z}\right) \\
& \frac{\partial I_{2}(x, y, z, t)}{\partial t}=\frac{\partial}{\partial x}\left[D_{I}(x, y, z, T) \frac{\partial I_{1}(x, y, z, t)}{\partial x}\right]+\frac{\partial}{\partial y}\left[D_{I}(x, y, z, T) \frac{\partial I_{1}(x, y, z, t)}{\partial y}\right]+ \\
& +\frac{\partial}{\partial z}\left[D_{I}(x, y, z, T) \frac{\partial I_{1}(x, y, z, t)}{\partial z}\right]-k_{I, V}(x, y, z, T)\left[\alpha_{2 I}+I_{1}(x, y, z, t)\right]\left[\alpha_{2 V}+V_{1}(x, y, z, t)\right]- \\
& -k_{I, I}(x, y, z, T)\left[\alpha_{2 I}+I_{1}(x, y, z, t)\right]^{2} \\
& \frac{\partial V_{2}(x, y, z, t)}{\partial t}=\frac{\partial}{\partial x}\left[D_{V}(x, y, z, T) \frac{\partial V_{1}(x, y, z, t)}{\partial x}\right]+\frac{\partial}{\partial y}\left[D_{V}(x, y, z, T) \frac{\partial V_{1}(x, y, z, t)}{\partial y}\right]+ \\
& +\frac{\partial}{\partial z}\left[D_{V}(x, y, z, T) \frac{\partial V_{1}(x, y, z, t)}{\partial z}\right]-k_{I, V}(x, y, z, T)\left[\alpha_{2 I}+I_{1}(x, y, z, t)\right]\left[\alpha_{2 V}+V_{1}(x, y, z, t)\right]- \\
& -k_{V, V}(x, y, z, T)\left[\alpha_{2 V}+V_{1}(x, y, z, t)\right]^{2} \\
& \frac{\partial \Phi_{I 2}(x, y, z, t)}{\partial t}=\frac{\partial}{\partial x}\left[D_{\Phi I}(x, y, z, T) \frac{\partial \Phi_{I 1}(x, y, z, t)}{\partial x}\right]+k_{I, I}(x, y, z, T) I^{2}(x, y, z, t)+ \\
& +\frac{\partial}{\partial y}\left[D_{\Phi I}(x, y, z, T) \frac{\partial \Phi_{I 1}(x, y, z, t)}{\partial y}\right]+\frac{\partial}{\partial z}\left[D_{\Phi I}(x, y, z, T) \frac{\partial \Phi_{I 1}(x, y, z, t)}{\partial z}\right]- \\
& -k_{I}(x, y, z, T) I(x, y, z, t)
\end{aligned}
$$


International Journal of Applied Control, Electrical and Electronics Engineering (IJACEEE) Vol 3, No.4, November 2015

$$
\begin{gathered}
\frac{\partial \Phi_{V 2}(x, y, z, t)}{\partial t}=\frac{\partial}{\partial x}\left[D_{\Phi V}(x, y, z, T) \frac{\partial \Phi_{V 1}(x, y, z, t)}{\partial x}\right]+k_{V, V}(x, y, z, T) V^{2}(x, y, z, t)+ \\
+\frac{\partial}{\partial y}\left[D_{\Phi V}(x, y, z, T) \frac{\partial \Phi_{V 1}(x, y, z, t)}{\partial y}\right]+\frac{\partial}{\partial z}\left[D_{\Phi V}(x, y, z, T) \frac{\partial \Phi_{V 1}(x, y, z, t)}{\partial z}\right]- \\
-k_{V}(x, y, z, T) V(x, y, z, t) .
\end{gathered}
$$

Integration of left and right sides of Eqs. (8)-(10) on time gives us possibility to obtain relations for the second-order approximations of the required concentrations in the final form

$$
\begin{aligned}
& C_{2}(x, y, z, t)=\frac{\partial}{\partial x}\left(\int_{0}^{t} D_{L}(x, y, z, T)\left[1+\varsigma_{1} \frac{V(x, y, z, \tau)}{V^{*}}+\varsigma_{2} \frac{V^{2}(x, y, z, \tau)}{\left(V^{*}\right)^{2}}\right] \frac{\partial C_{1}(x, y, z, \tau)}{\partial x} \times\right. \\
& \left.\times\left\{1+\xi \frac{\left[\alpha_{2 C}+C_{1}(x, y, z, \tau)\right]^{\gamma}}{P^{\gamma}(x, y, z, T)}\right\}\right) d \tau+\frac{\partial}{\partial y}\left(\int_{0}^{t}\left[1+\varsigma_{1} \frac{V(x, y, z, \tau)}{V^{*}}+\varsigma_{2} \frac{V^{2}(x, y, z, \tau)}{\left(V^{*}\right)^{2}}\right] \times\right. \\
& \left.\times D_{L}(x, y, z, T)\left\{1+\xi \frac{\left[\alpha_{2 C}+C_{1}(x, y, z, \tau)\right]^{\gamma}}{P^{\gamma}(x, y, z, T)}\right\} \frac{\partial C_{1}(x, y, z, \tau)}{\partial y} d \tau\right)+\frac{\partial}{\partial z}\left(\int_{0}^{t} \frac{\partial C_{1}(x, y, z, \tau)}{\partial z} \times\right. \\
& \left.\times D_{L}(x, y, z, T)\left[1+\varsigma_{1} \frac{V(x, y, z, \tau)}{V^{*}}+\varsigma_{2} \frac{V^{2}(x, y, z, \tau)}{\left(V^{*}\right)^{2}}\right] d \tau\right)+f_{C}(x, y, z) \\
& I_{2}(x, y, z, t)=\frac{\partial}{\partial x}\left[\int_{0}^{t} D_{I}(x, y, z, T) \frac{\partial I_{1}(x, y, z, \tau)}{\partial x} d \tau\right]+\frac{\partial}{\partial y}\left[\int_{0}^{t} D_{I}(x, y, z, T) \frac{\partial I_{1}(x, y, z, \tau)}{\partial y} d \tau\right]+ \\
& +\frac{\partial}{\partial z}\left[\int_{0}^{t} D_{I}(x, y, z, T) \frac{\partial I_{1}(x, y, z, \tau)}{\partial z} d \tau\right]-\int_{0}^{t} k_{I, I}(x, y, z, T)\left[\alpha_{2 I}+I_{1}(x, y, z, \tau)\right]^{2} d \tau- \\
& -\int_{0}^{t} k_{I, V}(x, y, z, T)\left[\alpha_{2 I}+I_{1}(x, y, z, \tau)\right]\left[\alpha_{2 V}+V_{1}(x, y, z, \tau)\right] d \tau+f_{I}(x, y, z) \\
& V_{2}(x, y, z, t)=\frac{\partial}{\partial x}\left[\int_{0}^{t} D_{V}(x, y, z, T) \frac{\partial V_{1}(x, y, z, \tau)}{\partial x} d \tau\right]+\frac{\partial}{\partial y}\left[\int_{0}^{t} D_{V}(x, y, z, T) \frac{\partial V_{1}(x, y, z, \tau)}{\partial y} d \tau\right]+ \\
& +\frac{\partial}{\partial z}\left[\int_{0}^{t} D_{V}(x, y, z, T) \frac{\partial V_{1}(x, y, z, \tau)}{\partial z} d \tau\right]-\int_{0}^{t} k_{V, V}(x, y, z, T)\left[\alpha_{2 I}+V_{1}(x, y, z, \tau)\right]^{2} d \tau- \\
& -\int_{0}^{t} k_{I, V}(x, y, z, T)\left[\alpha_{2 I}+I_{1}(x, y, z, \tau)\right]\left[\alpha_{2 V}+V_{1}(x, y, z, \tau)\right] d \tau+f_{V}(x, y, z) \\
& \Phi_{I 2}(x, y, z, t)=\frac{\partial}{\partial x}\left[\int_{0}^{t} D_{\Phi I}(x, y, z, T) \frac{\partial \Phi_{I 1}(x, y, z, \tau)}{\partial x} d \tau\right]-\int_{0}^{t} k_{I}(x, y, z, T) I(x, y, z, \tau) d \tau+ \\
& +\frac{\partial}{\partial y}\left[\int_{0}^{t} D_{\Phi I}(x, y, z, T) \frac{\partial \Phi_{I 1}(x, y, z, \tau)}{\partial y} d \tau\right]+\frac{\partial}{\partial z}\left[\int_{0}^{t} D_{\Phi I}(x, y, z, T) \frac{\partial \Phi_{I 1}(x, y, z, \tau)}{\partial z} d \tau\right]+ \\
& +\int_{0}^{t} k_{I, I}(x, y, z, T) I^{2}(x, y, z, \tau) d \tau+f_{\Phi I}(x, y, z)
\end{aligned}
$$




$$
\begin{gathered}
\Phi_{V 2}(x, y, z, t)=\frac{\partial}{\partial x}\left[\int_{0}^{t} D_{\Phi V}(x, y, z, T) \frac{\partial \Phi_{V 1}(x, y, z, \tau)}{\partial x} d \tau\right]-\int_{0}^{t} k_{V}(x, y, z, T) V(x, y, z, \tau) d \tau+ \\
+\frac{\partial}{\partial y}\left[\int_{0}^{t} D_{\Phi V}(x, y, z, T) \frac{\partial \Phi_{V 1}(x, y, z, \tau)}{\partial y} d \tau\right]+\frac{\partial}{\partial z}\left[\int_{0}^{t} D_{\Phi V}(x, y, z, T) \frac{\partial \Phi_{V 1}(x, y, z, \tau)}{\partial z} d \tau\right]+ \\
+\int_{0}^{t} k_{V, V}(x, y, z, T) V^{2}(x, y, z, \tau) d \tau+f_{\Phi V}(x, y, z) .
\end{gathered}
$$

We determine average values of the second-orders approximations of the required functions by the standard relation $[15,16]$

$$
\alpha_{2 \rho}=\frac{1}{\Theta L_{x} L_{y} L_{z}} \int_{0}^{\Theta} \int_{0}^{L_{x} L_{y} L_{z}} \int_{0}^{0}\left[\rho_{0}(x, y, z, t)-\rho_{1}(x, y, z, t)\right] d z d y d x d t
$$

Substitution of the relations $(8 a)-(10 a)$ into the relation (11) gives us possibility to obtain relations for the required average values $\alpha_{2 \rho}$

$$
\begin{aligned}
& \alpha_{2 C}=\frac{1}{L_{x} L_{y} L_{z}} \int_{0}^{L_{x}} \int_{0}^{L_{y} L_{z}} \int_{0} f_{C}(x, y, z) d z d y d x \\
& \alpha_{2 I}=\frac{1}{2 A_{I I 00}}\left\{\left(1+A_{I V 01}+A_{I I 10}+\alpha_{2 V} A_{I V 00}\right)^{2}-4 A_{I I 00}\left[\alpha_{2 V} A_{I V 10}-A_{I I 20}+A_{I V 11}-\right.\right. \\
& \left.\left.-\frac{1}{L_{x} L_{y} L_{z}} \int_{0}^{L_{Y}} \int_{0}^{L_{y} L_{z}} \int_{0} f_{I}(x, y, z) d z d y d x\right]\right\}^{\frac{1}{2}}-\frac{1+A_{I V 01}+A_{I I 10}+\alpha_{2 V} A_{I V 00}}{2 A_{I I 00}} \\
& \alpha_{2 V}=\frac{1}{2 B_{4}} \sqrt{\frac{\left(B_{3}+A\right)^{2}}{4}-4 B_{4}\left(y+\frac{B_{3} y-B_{1}}{A}\right)}-\frac{B_{3}+A}{4 B_{4}} .
\end{aligned}
$$

Parameters $A_{a b i j}$ and other parameters in the relations (13) are presented in the Appendix. Parameters $\alpha_{a b i j}$ and other parameters in the relations (13) could be written as

$$
\begin{aligned}
& \alpha_{2 \Phi_{I}}=A_{I I 20}-\frac{1}{\Theta L_{x} L_{y} L_{z}} \int_{0}^{\Theta}(\Theta-t) \int_{0}^{L_{x} L_{y} \int_{0} \int_{0}} k_{0} k_{I}(x, y, z, T) I(x, y, z, t) d z d y d x d t+ \\
& +\frac{1}{L_{x} L_{y} L_{z}} \int_{0}^{L_{x} L_{y} L_{z}} \int_{0} \int_{0} f_{\Phi I}(x, y, z) d z d y d x \\
& \alpha_{2 \Phi_{V}}=A_{V V 20}-\frac{1}{\Theta L_{x} L_{y} L_{z}} \int_{0}^{\Theta}(\Theta-t) \int_{0}^{L_{x} L_{y} L_{z}} \int_{0} \int_{0} k_{V}(x, y, z, T) V(x, y, z, t) d z d y d x d t+ \\
& +\frac{1}{L_{x} L_{y} L_{z}} \int_{0}^{L_{x}} \int_{0}^{L_{y} L_{z}} \int_{0} f_{\Phi V}(x, y, z) d z d y d x .
\end{aligned}
$$

The considered substitution gives us possibility to obtain equation for parameter $\alpha_{2 C}$. Solution of the equation depends on value of parameter $\gamma$. Analysis of spatio- temporal distributions of concentrations of dopant and radiation defects has been done by using their second-order approximations framework method of averaging of function corrections with decreased quantity of iterative 
steps. The second-order approximation is usually a sufficient approximation to obtain qualitative and some quantitative results. Results of analytical modeling have been checked numerically.

\section{DISCUSSION}

By using the calculated in the previous section relations we analyzed dynamic of redistribution of dopant with account redistribution of radiation defects. Figs. 2 show typical distributions of concentrations of infused and implanted dopants for this case, when dopant diffusion coefficient in the epitaxial layer is larger, than in the substrate. The figures show, that interface between materials gives a possibility to increase sharpness of $p-n$-junction and at the same time to increase homogeneity of concentration of dopant in doped area.

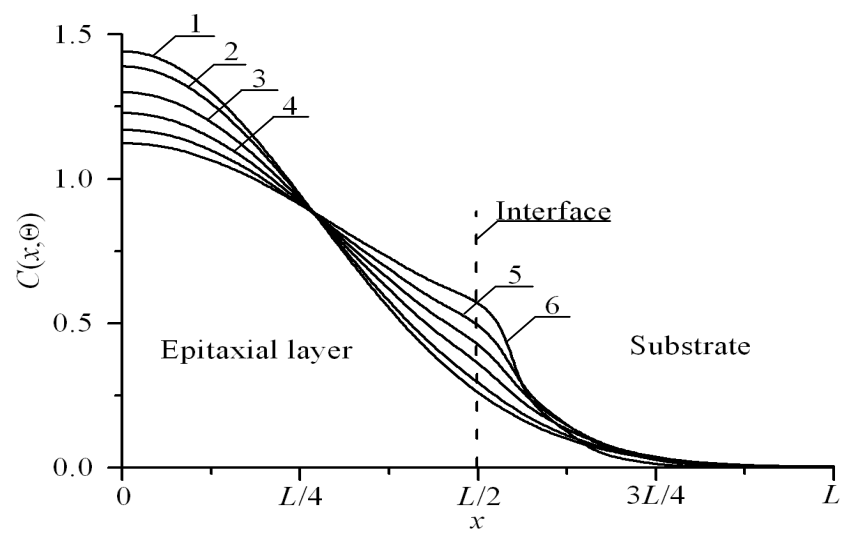

Fig. 2a. Distributions of concentrations of infused dopant in heterostructure from Fig. 1 in direction, which is perpendicular to interface between layers of heterostructure. Increasing of number of curves corresponds to increasing of difference between values of dopant diffusion coefficient in layers of heterostructure. The curves have been calculated under condition, when dopant diffusion coefficient in doped layer is larger, than in nearest layer

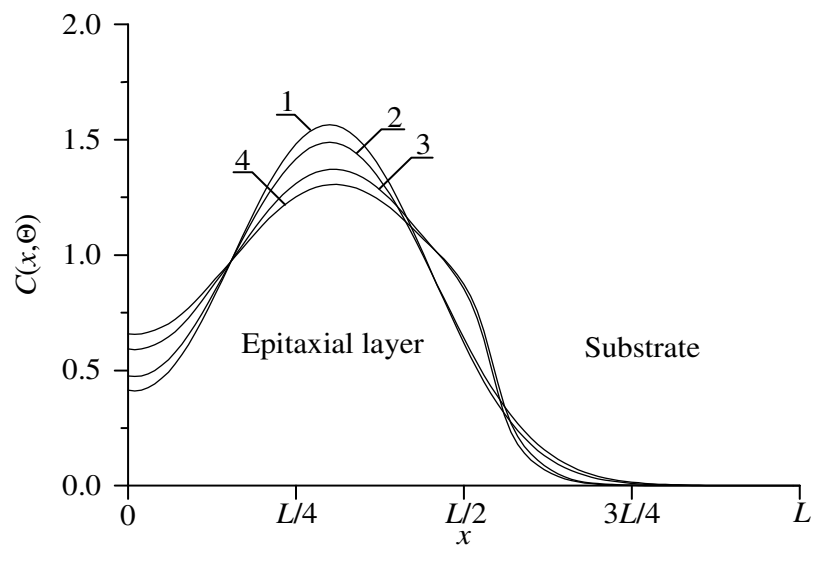

Fig. $2 b$. Spatial distributions of implanted dopant concentration after annealing. Curves 1 and 2 are calculated distributions of dopant concentration in homogenous structure for two values of annealing time. Curves 3 and 4 are calculated distributions of dopant concentration in heterostructure for the same values of annealing time under condition, when dopant diffusion coefficient in doped layer is larger, than in nearest layer 
It is known, that increasing of annealing time leads to increasing of diffusion length into nearest materials of heterostructure. Decrease of annealing time does not allow to fully doped section of the epitaxial layer. We determine compromise annealing time framework recently introduced criterion [17-22]. Framework the criterion we approximate real distribution of concentration of dopant by the step-wise function $\psi(x, y, z)$ (see Figs. 3)

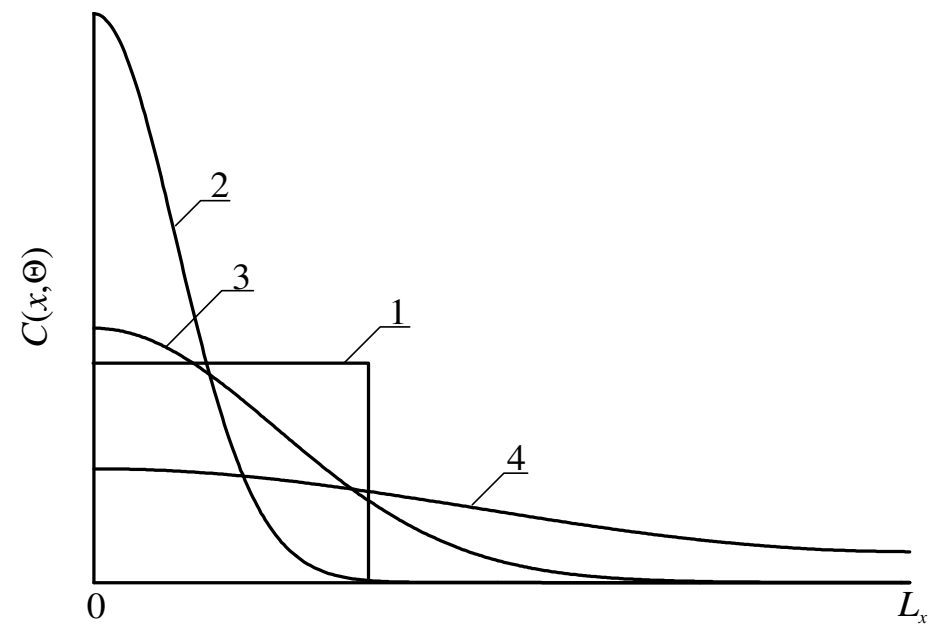

Fig.3a. Spatial distributions of concentration of implanted dopant in heterostructure from Fig. 1. Curve 1 is the idealized distribution of dopant. Curves 2-4 are the real distributions of dopant for different values of annealing time. Increasing of number of curve corresponds to increasing of annealing time

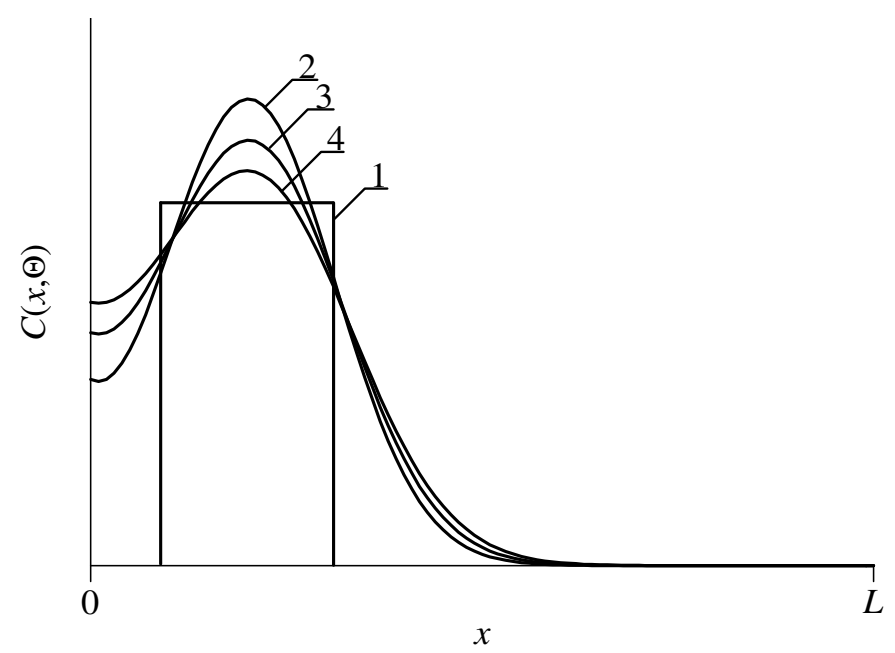

Fig.3b. Spatial distributions of concentration of infused dopant in heterostructure from Fig. 1. Curve 1 is the idealized distribution of dopant. Curves 2-4 are the real distributions of dopant for different values of annealing time. Increasing of number of curve corresponds to increasing of annealing time

$$
U=\frac{1}{L_{x} L_{y} L_{z}} \int_{0}^{L_{x}} \int_{0}^{L_{y}} \int_{0}^{L_{z}}[C(x, y, z, \Theta)-\psi(x, y, z)] d z d y d x
$$

Distributions of concentrations of dopants in Figs. 2 corresponds exactly to compromise annealin time. It should be noted, that presents of interface between layers of heterostructure gives us possibility to manufacture more thin heterotransistor. 


\section{CONCLUSIONS}

In this paper we introduce an approach to manufacture double-base heterotransistor. Several recommendations for optimization of diffusion and ion types of doping to decrease dimensions of double-base heterotransistor have been formulated.

\section{ACKNOWLEDGEMENTS}

This work is supported by the agreement of August 27, 2013 № 02.B.49.21.0003 between The Ministry of education and science of the Russian Federation and Lobachevsky State University of Nizhni Novgorod and educational fellowship for scientific research of Government of Russian and of Nizhny Novgorod State University of Architecture and Civil Engineering.

\section{REFERENCES}

[1] A.A. Andronov, N.T. Bagraev, L.E. Klyachkin, A.M. Malyarenko, S.B. Robozerov. Ultrashallow p+n-junction in silicon (111): electron-beam diagnostics of nearsurface region. Semiconductors. Vol. 33 (1). P. 58-63 (1999).

[2] M. Nichterwitz, R. Caballero, C.A. Kaufmann, H.-W. Schock, T. Unold. Generation-dependent charge carrier transport in $\mathrm{Cu}(\mathrm{In}, \mathrm{Ga}) \mathrm{Se} 2 / \mathrm{CdS} / \mathrm{ZnO}$ thin-film solar-cells. J. Appl. Phys. Vol. 113 (16). P. 044515-044530 (2013).

[3] J.Y. Fang, G.Y. Lee, J.I. Chyi, C.P. Hsu, Y.W. Kang, K.C. Fang, W.L. Kao, D.J. Yao, C.H. Hsu, Y.F. Huang, C.C. Chen, S.S. Li, J.A. Yeh, F. Ren, Y.L. Wang. Viscosity-dependent drain current noise of AlGaN/GaN high electron mobility transistor in polar liquids. J. Appl. Phys. Vol. 114 (20). P. 204503-204507 (2013).

[4] G. Volovich. Modern chips UM3Ch class D manufactured by firm MPS. Modern Electronics. Issue 2. P. 10-17 (2006).

[5] A. Kerentsev, V. Lanin. Constructive-technological features of MOSFET-transistors. Power Electronics. Issue 1. P. 34-38 (2008).

[6] O.A. Ageev, A.E. Belyaev, N.S. Boltovets, V.N. Ivanov, R.V. Konakova, Ya.Ya. Kudryk, P.M. Lytvyn, V.V. Milenin, A.V. Sachenko. Influence of displacement of the electron-hole equilibrium on the process of transition metals diffusion in GaAs. Semiconductors. Vol. 43 (7). P. 897-903 (2009).

[7] N.I. Volokobinskaya, I.N. Komarov, T.V. Matioukhina, V.I. Rechetniko, A.A. Rush, I.V. Falina, A.S. Yastrebov. Investigation of technological processes of manufacturing of the bipolar power highvoltage transistors with a grid of inclusions in the collector region. Semiconductors. Vol. 35 (8). P. 1013-1017 (2001).

[8] K.K. Ong, K.L. Pey, P.S. Lee, A.T.S. Wee, X.C. Wang, Y.F. Chong. Dopant distribution in the recrystallization transient at the maximum melt depth induced by laser annealing. Appl. Phys. Lett. 89 (17), 172111-172114 (2006).

[9] H.T. Wang, L.S. Tan, E. F. Chor. Pulsed laser annealing of Be-implanted GaN J. Appl. Phys. 98 (9), 094901-094905 (2006).

[10] Yu.V. Bykov, A.G. Yeremeev, N.A. Zharova, I.V. Plotnikov, K.I. Rybakov, M.N. Drozdov, Yu.N. Drozdov, V.D. Skupov. Diffusion processes in semiconductor structures during microwave annealing. Radiophysics and Quantum Electronics. Vol. 43 (3). P. 836-843 (2003).

[11] V.V. Kozlivsky. Modification of semiconductors by proton beams (Nauka, Sant-Peterburg, 2003, in Russian).

[12] V.L. Vinetskiy, G.A. Kholodar', Radiative physics of semiconductors. ("Naukova Dumka", Kiev, 1979, in Russian).

[13] Z.Yu. Gotra. Technology of microelectronic devices (Radio and communication, Moscow, 1991).

[14] P.M. Fahey, P.B. Griffin, J.D. Plummer. Point defects and dopant diffusion in silicon. Rev. Mod. Phys. 1989. V. 61. № 2. P. 289-388.

[15] Yu.D. Sokolov. About the definition of dynamic forces in the mine lifting. Applied Mechanics. Vol.1 (1). P. 23-35 (1955). 
[16] E.L. Pankratov. Dynamics of delta-dopant redistribution during heterostructure growth. The European Physical Journal B. 2007. V. 57, №3. P. 251-256.

[17] E.L. Pankratov. Dopant Diffusion Dynamics and Optimal Diffusion Time as Influenced by DiffusionCoefficient Nonuniformity. Russian Microelectronics. 2007. V.36 (1). P. 33-39.

[18] E.L. Pankratov. Redistribution of dopant during annealing of radiative defects in a multilayer structure by laser scans for production an implanted-junction rectifiers.Int. J. Nanoscience. Vol. 7 (4-5). P. 187-197 (2008).

[19] E.L. Pankratov. Decreasing of depth of implanted-junction rectifier in semiconductor heterostructure by optimized laser annealing. J. Comp. Theor. Nanoscience. Vol. 7 (1). P. 289-295 (2010).

[20] E.L. Pankratov, E.A. Bulaeva. Application of native inhomogeneities to increase compactness of vertical field-effect transistors. J. Comp. Theor. Nanoscience. Vol. 10 (4). P. 888-893 (2013).

[21] E.L. Pankratov, E.A. Bulaeva. An approach to manufacture of bipolar transistors in thin film structures. On the method of optimization. Int. J. Micro-Nano Scale Transp. Vol. 4 (1). P. 17-31 (2014).

[22] E.L. Pankratov, E.A. Bulaeva. Increasing of sharpness of diffusion-junction heterorectifier by using radiation processing. Int. J. Nanoscience. Vol. 11 (5). P. 1250028-1250035 (2012).

\section{Authors:}

Pankratov Evgeny Leonidovich was born at 1977. From 1985 to 1995 he was educated in a secondary school in Nizhny Novgorod. From 1995 to 2004 he was educated in Nizhny Novgorod State University: from 1995 to 1999 it was bachelor course in Radiophysics, from 1999 to 2001 it was master course in Radiophysics with specialization in Statistical Radiophysics, from 2001 to 2004 it was PhD course in Radiophysics. From 2004 to 2008 E.L. Pankratov was a leading technologist in Institute for Physics of Microstructures. From 2008 to 2012 E.L. Pankratov was a senior lecture/Associate Professor of Nizhny Novgorod State University of Architecture and Civil Engineering. Now E.L. Pankratov is in his Full Doctor course in Radiophysical Department of Nizhny Novgorod State University. He has 110 published papers in area of his researches.

Bulaeva Elena Alexeevna was born at 1991. From 1997 to 2007 she was educated in secondary school of village Kochunovo of Nizhny Novgorod region. From 2007 to 2009 she was educated in boarding school "Center for gifted children". From 2009 she is a student of Nizhny Novgorod State University of Architecture and Civil Engineering (spatiality "Assessment and management of real estate"). At the same time she is a student of courses "Translator in the field of professional communication" and "Design (interior art)" in the University. E.A. Bulaeva was a contributor of grant of President of Russia (grant № MK-548.2010.2). She has 74 published papers in area of her researches.

\section{APPENDIX}

$$
\begin{aligned}
& A_{a b i j}=\frac{1}{\Theta L_{x} L_{y} L_{z}} \int_{0}^{\Theta}(\Theta-t) \int_{0}^{L_{x}} \int_{0}^{L_{y} L_{z}} \int_{0} k_{a, b}(x, y, z, T) I_{1}^{i}(x, y, z, t) V_{1}^{j}(x, y, z, t) d z d y d x d t, \\
& B_{4}=A_{I V 00}^{2} A_{I V 00}^{2}-2\left(A_{I V 00}^{2}-A_{I I 00} A_{V V 00}\right)^{2}, B_{3}=A_{I V 00} A_{I V 00}^{2}+A_{I V 01} A_{I V 00}^{3}+A_{I V 00} A_{I I 10} A_{I V 00}^{2}- \\
& -4\left(A_{I V 00}^{2}-A_{I I 00} A_{V V 00}\right)\left[2 A_{I V 01} A_{I V 00}+2 A_{I V 00}\left(1+A_{I V 01}+A_{I I 10}\right)-2 A_{I I 00}\left(A_{I V 10}+A_{V V 10}+1\right)\right]- \\
& -4 A_{I V 10} A_{I V 10} A_{I I 00} A_{I V 00}^{2}+2 A_{I V 00} A_{I V 01} A_{I V 00}^{2}, B_{2}=A_{I V 00}^{2}\left\{\left(1+A_{I V 01}+A_{I I 10}\right)^{2}+A_{I V 00}^{2} A_{I V 01}^{2}-A_{I I 00} \times\right. \\
& \times 4\left[A_{I V 11}-A_{I I 20}-\frac{1}{L_{x} L_{y} L_{z}} \int_{0}^{L_{x}} \int_{0}^{L_{y} L_{z}} \int_{0} f_{I}(x, y, z) d z d y d x\right]+2 A_{I V 00} A_{I V 01}\left(A_{I V 00}+A_{I V 00} A_{I V 01}+\right. \\
& \left.\left.+A_{I V 00} A_{I I 10}-4 A_{I V 10} A_{I I 00}\right)\right\}\left[2 A_{I V 01} A_{I V 00}+2 A_{I V 00}\left(1+A_{I V 01}+A_{I I 10}\right)-2 A_{I I 00}\left(A_{I V 10}+A_{V V 10}+\right.\right. \\
& +1)]^{2}+2\left[A_{I V 01}\left(1+A_{I V 01}+A_{I I 10}\right)+\frac{2}{L_{x} L_{y} L_{z}} \int_{0}^{L_{x}} \int_{0}^{L_{y} L_{z}} \int_{0} f_{V}(x, y, z) d z d y d x-2\left(A_{V V 20}-A_{I V 11}\right) \times\right. \\
& \left.\times A_{I I 00}+A_{I V 01}\left(1+A_{I V 01}+A_{I I 10}\right)\right]\left[2 A_{I V 00}\left(1+A_{I V 01}+A_{I I 10}\right)-2 A_{I I 00}\left(A_{I V 10}+A_{V V 10}+1\right)+\right.
\end{aligned}
$$


International Journal of Applied Control, Electrical and Electronics Engineering (IJACEEE) Vol 3, No.4, November 2015

$$
\begin{aligned}
& \left.\left.+2 A_{I V 01} A_{I V 00}\right]\right\}, B_{1}=2 A_{I V 00} A_{I V 01}\left(1+A_{I V 01}+A_{I I 10}\right)^{2}-8 A_{I V 00} A_{I V 01} A_{I I 00}\left[A_{I V 11}-A_{I I 20}-\right. \\
& \left.-\frac{1}{L_{x} L_{y} L_{z}} \int_{0}^{L_{x} L_{y} L_{0}} \int_{0} \int_{0}^{z} f_{I}(x, y, z) d z d y d x\right]+\left(A_{I V 00}+A_{I V 00} A_{I V 01}+A_{I V 00} A_{I I 10}-4 A_{I V 10} A_{I I 00}\right) \times \\
& \times A_{I V 01}^{2}-2\left[\frac{2 A_{I I 00}}{L_{x} L_{y} L_{z}} \int_{0}^{L_{x}} \int_{0}^{L_{y} L_{z}} \int_{0} f_{I}(x, y, z) d z d y d x+A_{I V 01}\left(1+A_{I V 01}+A_{I I 10}\right)-\left(A_{V V 20}-A_{I V 11}\right) \times\right. \\
& \left.\times 2 A_{I I 00}+A_{I V 01}\left(1+A_{I V 01}+A_{I I 10}\right)\right]\left[2 A_{I V 00}\left(1+A_{I V 01}+A_{I I 10}\right)-2 A_{I I 00}\left(A_{I V 10}+A_{V V 10}+1\right)+\right. \\
& \left.+2 A_{I V 01} A_{I V 00}\right], B_{0}=4 A_{I I 00} A_{I V 01}^{2}\left[A_{I I 20}+\frac{1}{L_{x} L_{y} L_{z}} \int_{0}^{L_{x} L_{y} L_{0}} \int_{0}^{0} f_{0} f_{I}(x, y, z) d z d y d x-A_{I V 11}\right]- \\
& -\left[\frac{2 A_{I I 00}}{L_{x} L_{y} L_{z}} \int_{0}^{L_{x}} \int_{0}^{L_{2} L_{z}} \int_{0} f_{V}(x, y, z) d z d y d x+A_{I V 01}\left(1+A_{I V 01}+A_{I I 0}\right)-2 A_{I I 00}\left(A_{V V 20}-A_{I V 11}\right)+\right. \\
& \left.+A_{I V 01}\left(1+A_{I V 01}+A_{I I 10}\right)\right]^{2}+A_{I V 01}^{2}\left(A_{I V 01}+A_{I I 10}+1\right)^{2}, \quad y=\sqrt[3]{\sqrt{q^{2}+p^{3}}-q}+B_{2} / 6- \\
& -\sqrt[3]{\sqrt{q^{2}+p^{3}}+q}, q=\frac{1}{8}\left[\frac{B_{2}^{3}}{27}+B_{0}\left(4 B_{2}-B_{3}^{2}\right)-B_{1}^{2}+\frac{B_{2}}{6}\left(2 B_{1} B_{3}-8 B_{0}\right)\right], A=\sqrt{8 y+B_{3}^{2}-4 B_{2}} \text {, } \\
& p=\left[3\left(2 B_{1} B_{3}-8 B_{0}\right)-2 B_{2}^{2}\right] / 72 \text {. }
\end{aligned}
$$

